\section{Dae-Chul Cho ${ }^{1, *}$, Kyoungsu Ryu ${ }^{2, *}$, Kyoung-Tae $\mathrm{Kim}^{1}$, Joo-Kyung Sung ${ }^{1}$}

${ }^{1}$ Department of Neurosurgery, Kyungpook National University School of Medicine, Daegu, 'Department of Neurosurgery, Halla Hospital, Jeju, Korea

Corresponding Author: Dae-Chul Cho

Department of Neurosurgery, Kyungpook National University Hospital, 130 Dongdeok-ro, Jung-gu, Daegu 41944, Korea

Tel: +82-53-420-5649

Fax: +82-53-423-0504

E-mail: dccho@knu.ac.kr

${ }^{*}$ These authors contributed equally to this study and should be considered co-first authors.

Received: April 7, 2017

Revised: June 20, 2017

Accepted: June 22, 2017

\title{
The Therapeutic Effects of Combination Therapy with Curcumin and Alendronate on Spine Fusion Surgery in the Ovariectomized Rats
}

Objective: The purpose of this study was to evaluate the therapeutic effects of combination therapy with curcumin and alendronate on spine fusion surgery in ovariectomized rats.

Methods: Thirty-two female Sprague-Dawley rats (12 weeks old) underwent bilateral ovariectomy (OVX). Eight weeks after surgery, animals underwent intertransverse spine fusion at L4-5. The rats were randomly distributed amongst 4 groups; untreated OVX group, curcumin administered group, alendronate administered group, and the combination therapy group. At 8 weeks after fusion surgery, the animals were sacrificed and the fusion mass was assessed by manual palpation, radiographic scan, and micro-computed tomographic scan. In addition, mechanical strength was determined by a 3-point bending test.

Results: Based on the results of manual palpation testing and 3-dimensional micro-computed tomography scanning, solid bone fusion rate was 50\% (4 of 8 ) in the OVX group, $75 \%$ (6 of 8 ) in the alendronate-only and curcumin-only group, and $87.5 \%$ (7 of 8 ) in the combination therapy group, respectively. The combination therapy group had a higher fusion rate compared with the other treatment groups, though not statistically significantly $(p>0.05)$. And the combination therapy group had a significant increase in fusion volume at 8 weeks after spine fusion surgery compared with curcumin-only group $(p=0.039)$. The 3-point bending test showed that combination therapy group had a significantly greater maximal load value compared to that of curcumin-only group $(p=0.024)$.

Conclusion: The present study demonstrated that additional treatment of curcumin and alendronate after spine fusion surgery in rat can promote higher fusion volume, and improve bone mechanical strength.

Key Words: Bone fusion, Curcumin, Osteoporosis, Ovariectomy, Rat

\section{INTRODUCTION}

Geriatric populations, who are the potential candidates of osteoporosis, are increasing worldwide, with an associated increase in the number of individuals undergoing pharmacotherapy to prevent or treat osteopososis ${ }^{20)}$. Thus, osteoporosis is an important worldwide public health concern ${ }^{2,8,20)}$. There are 2 kinds of treatment options for osteoporosis: antiresorptive therapy, which inhibits bone resorption, and anabolic therapy, which increases bone formation $^{8,10,21,25)}$. Bisphosphonates, which are potent antiresorptive agents, have been widely used for clinical treatment of osteoporosis, $6,18,21,25)$. Due to the theoretical action of bisphosphonates binding to bone at sites of active bone remodeling, treatment with bisphosphonates can modulate the remodeling phase in fracture repair and bone fusion ${ }^{26)}$.

Spinal instrumentation and fusion is one of the important surgical options for spine diseases, so a great many elderly persons with osteoporosis may require spine fusion ${ }^{16,17)}$. However, osteoporosis causes a difficult environment for spinal fusion surgery in patients with a degenerative spinal disease or traumatic fracture ${ }^{7,9)}$.

Because many patients who need spine fusion also suffer from osteoporosis, they require concurrent antiosteoporotic therapy, including bisphosphonate $^{16}$. Although several studies have reported the influence of bisphosphonate treatment on spine fusion in animal and human studies, the role of bisphosphonate on bone fusion after spine fusion surgery remains controversial $^{3,16-19,24,26)}$.

Curcumin is found in the rhizomes of the turmeric plant (Curcumin longa L.), a perennial herb belonging to the ginger family cultivated extensively in South and Southeast tropical Asia ${ }^{14)}$. Recent studies have reported the antiresorptive effects of curcumin on bone re 
modeling, which is actually similar to the actions of the bisphosphonates ${ }^{411,12,14)}$. French et al. ${ }^{12)}$ studied the long-term effects of curcumin administration in aging ovariectomized rats. They concluded that curcumin produced beneficial changes in bone turnover and an increase in bone strength in the ovariectomized mature rat model of postmenopausal osteoporosis. At our institute, we investigated therapeutic advantages of treatment of highdose curcumin in the ovariectomized rat ${ }^{5}$. In addition, we demonstrated a synergistic bone sparing effect of curcumin and alendronate in ovariectomized rat ${ }^{6}$.

However, to our knowledge, no controlled studies have been undertaken to determine whether curcumin promotes bone fusion and mechanical strength in spinal fusion surgery.

Therefore, the purpose of this study was to evaluate the therapeutic effects of combination therapy with curcumin and alendronate on spinal bone fusion in ovariectomized rats. We assessed bone fusion rates, the fusion volume using three-dimensional microcomputed tomography (3D- $\mu \mathrm{CT})$, and bone mechanical strength in ovariectomized rats.

\section{MATERIALS AND METHODS}

\section{Animals, Ovariectomy and Spine Fusion Surgery}

All experiments involving animals were performed in accordance with the animal care guidelines issued by the National Institutes of Health, and were approved by the Institutional Animal Care Committee at Kyungpook National University.

Thirty-two female Sprague-Dawley rats (11 weeks old) were purchased from Samtako Bio Inc. (Osan, Korea) and acclimated to the laboratory conditions for 1 week before the experiment. The rats were housed in an air-conditioned room (relative humidity $45 \%-65 \%$ ) under a 12 -hour light/dark cycle at $22^{\circ} \mathrm{C} \pm 2{ }^{\circ} \mathrm{C}$ and given free access to food and tap water.

The following week (at 12 weeks of age), they underwent bilateral ovariectomy to induce osteoporosis, which was described in detail in our previous studies ${ }^{5-7}$. Subsequently 8 weeks after ovariectomy, autologous spinal bone fusion surgery was performed in all ovariectomized rats. Briefly, the rats were anesthetized intraperitoneally with a mixture of xylazine $(10 \mathrm{mg} / \mathrm{kg})$ and ketamine $(90 \mathrm{mg} / \mathrm{kg})$. After the surgical site was shaved and prepared, the rats were placed prone on the operating table. An L4-5 posterolateral intertransverse and translaminar arthrodesis was performed, as described in previous studies ${ }^{1,7}$. A posterior midline skin incision was made over the lumbar spine. The laminar-transverse process of the L4 and L5 vertebrae was exposed by dissecting the back muscles. Once exposed, the laminar-transverse process of the L4-5 vertebrae was decorticated with an electric drill until a blush of cancellous bone was observed. A total $300 \mathrm{mg}$ of autologous bone was harvested from both iliac crests through fascial incisions. The harvested iliac bone was morselized and implanted bilaterally on the decorticated fusion beds to bridge the L4 and L5 interlaminar and intertransverse space. Postoperative antibiotics were given subcutaneously (gentamicin, $0.5 \mathrm{mg} / \mathrm{kg}$ ).
After fusion surgery, the 32 ovariectomized animals, which had undergone spinal fusion surgery, were randomly distributed amongst 4 groups. The first eight animals constituted group 1 (untreated OVX group), groups 2 and 3 animals received curcumin therapy and alendronate therapy, respectively, and group 4 received combination therapy with curcumin and alendronate.

The bisphosphonate selected for administration was alendronate which has been shown to enhance bone microstructure and strength in ovariectomized rats ${ }^{15}$. Alendronate (Sigma-Aldrich Canada Ltd., Oakville, ON, Canada) was dissolved in phosphate-buffered saline at a volume of $2 \mathrm{~mL}$ and administered by a stomach tube (po) each week at a concentration of $35 \mu \mathrm{g} / \mathrm{kg}$ for 8 weeks.

Curcumin was administered orally by addition to phytoestrogen reduced animal diets. Dietary intakes were limited to $20 \mathrm{~g}$ per day in order to avoid excessive body weight gains following ovariectomy. All rats were fed on the control (AIN-76A) diet or a diet containing curcumin $50 \mathrm{mg} / \mathrm{kg} / \mathrm{day}$. In order to estimate curcumin dosage, the number of food pellets remaining after each animal was fed was recorded. Throughout the experimental period, the body weight was monitored once a week.

\section{Fusion Assessment}

Eight weeks after fusion surgery, the rats were sacrificed, and the L4-5 segments were removed. Fusion at the bone-grafted

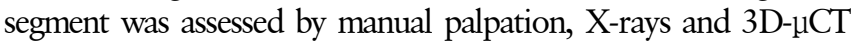
scanning at the L4-5 segment, as described by previous studies $^{1,7}$. Briefly, the harvested lumbar spine was palpated gently, and lateral side-bending motion was checked at the fused L4-5 level, and the adjacent levels above (L3-4) and below (L5-6). Two independent neurosurgeons blinded to the grouping of animals, tested the stability of lateral side-bending motion at the operative spinal segment.

A radiograph of each specimen was taken with a soft radiograph devise (M-60; SOFTEX Co. Ltd., Tokyo, Japan). Imaging was performed in the anteroposterior plane at $5 \mathrm{~mA}, 30 \mathrm{kV}$, for 130 seconds. $\mathrm{LCT}$ scanning (eXplore Locus SP, GE Healthcare, Chicago, IL, USA) of the lumbar spine was performed under consistent conditions. The formation of bridging bone between the L4-5 laminar-transverse space and consolidation of the grafted bone were evaluated.

Based on the results of manual palpation testing and 3D- $\mu \mathrm{CT}$ scanning, each specimen was classified as a solid union when no motion was observed by both neurosurgeons and bony continuity between the L4 and L5 laminar-transverse process was observed, and as a nonunion when motion was detected or discontinuity was observed by both neurosurgeons ${ }^{7,27}$.

\section{Fusion Volume Analysis}

Bone morphometric parameters of the fusion mass in the L4 and $\mathrm{L} 5$ vertebrae were assessed using $\mu \mathrm{CT}$. The scanning protocol was set at X-ray energy settings of $80 \mathrm{kV}$ and $80 \mu \mathrm{A}$, and 
the samples were scanned over one entire $360^{\circ}$ rotation, with an exposure time of $3,000 \mathrm{msec} /$ frame. An isotopic resolution of 15- to 40-um voxel size that displayed the microstructure of the rat lumbar vertebra was selected, and the angle of increment around the sample was set to $0.4^{\circ}$, which resulted in the acquisition of 900 2-dimensional images. For the bone analysis, bone tissue from the region of fusion mass in the L4-5 vertebrae was selected as the region of interest. Image information was obtained based on the automatic domain values produced by the computer. Bone volume fraction was applied to perform quantitative analysis using software provided with the $2.0+$ ABA Microview of the micro-CT system.

\section{Mechanical Strength Test}

Mechanical spinal strength was determined by a 3-point bending test. Each bone was positioned on the 2 lower supports of the anvil of a Universal Testing Machine (Instron 4202; Instron, Canton, MA, USA). Load was applied to the midportion of the fusion mass in the L4-5 vertebrae using a crosshead speed of $1.5 \mathrm{~mm} / \mathrm{min}$ for all tests. The load versus displacement data were recorded automatically by the Instron software (Instron series IX Automated Materials Tester, version 8.04.00), which subsequently calculated the mechanical parameters from the loaddisplacement curves.

\section{Statistical Analysis}

All statistical comparisons were made using SPSS ver. 17.0
(SPSS Inc., Chicago, IL, USA). Data are expressed as means \pm standard errors. Repeated measure 1-way analysis of variance was used to compare body weights between the study groups. Differences between groups in fusion status were analyzed using the chi-square test. The 1-way analysis of variance was used to identify significant differences in fusion volume and mechanical strength between the groups, and $\mathrm{p}$-values of $\leq 0.05$ were considered significant.

\section{RESULTS}

\section{Body Weights}

Body weights were measured once a week throughout the 16-week experiment period. Changes in the mean body weights between the groups over time are illustrated in Table 1 . The mean body weights in all groups were similar throughout the experiment period $(\mathrm{p}>0.05)$.

\section{Fusion Assessment}

Fig. 1 shows the representative X-ray images of the fused lumbar spine in all groups. The combination therapy group had larger fusion masses compared to those of other groups. The solid fusion rate was 50\% (4 of 8 ) in the OVX group, $75 \%(6$ of 8 ) in the alendronate only and curcumin only group, and $87.5 \%$ ( 7 of 8 ) in the combination therapy group, respectively. The fusion rate was not significantly different between experiment groups $(\mathrm{p}>0.05)$.

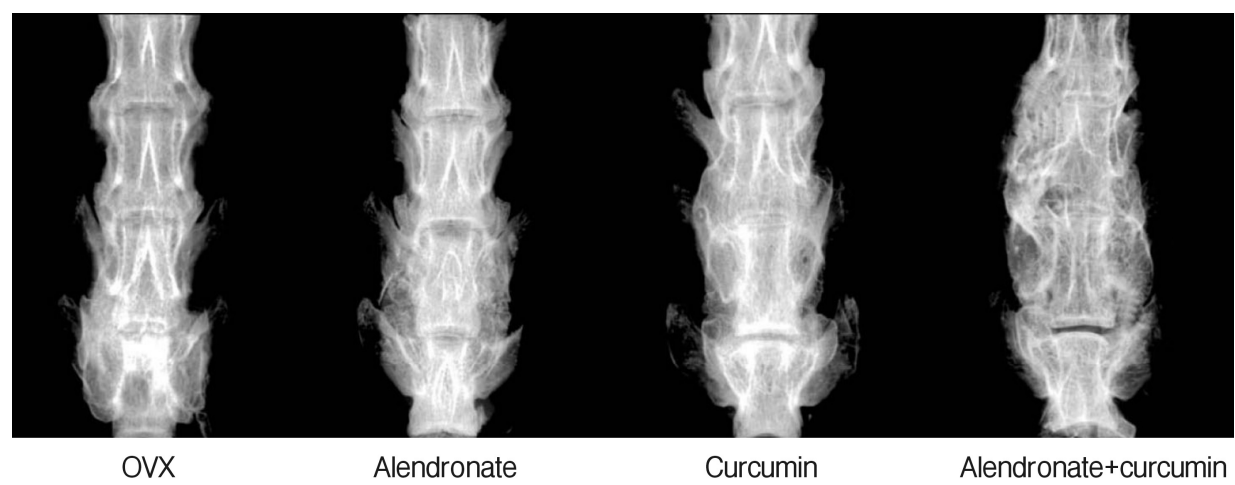

Fig 1. Representative posteroanterior radiographs of the spine in the ovariectomy (OVX) and experimental groups. The combination therapy group had larger fusion masses compared than other groups.

Table 1 . Mean body weights (g) of the rats

\begin{tabular}{|c|c|c|c|c|c|}
\hline \multirow{2}{*}{ Group } & \multicolumn{2}{|c|}{ Ovariectomy } & \multirow{2}{*}{$\begin{array}{c}\text { Fusion surgery } \\
8 \mathrm{Wk} \\
\end{array}$} & \multicolumn{2}{|c|}{ After fusion surgery } \\
\hline & $0 \mathrm{Wk}$ & $4 \mathrm{Wk}$ & & $8+4 \mathrm{Wk}$ & $8+8 \mathrm{Wk}$ \\
\hline Ovariectomy & $235.80 \pm 2.23$ & $336.00 \pm 4.84$ & $384.00 \pm 4.04$ & $387.63 \pm 6.64$ & $409.125 \pm 6.46$ \\
\hline Alendronate & $235.57 \pm 3.07$ & $348.67 \pm 3.77$ & $365.33 \pm 3.67$ & $370 \pm 2.39$ & $400 \pm 5.07$ \\
\hline Curcumin & $235.87 \pm 2.51$ & $340.67 \pm 2.86$ & $375.77 \pm 5.98$ & $361.88 \pm 3.58$ & $406.25 \pm 3.95$ \\
\hline Alendronate + curcumin & $236.53 \pm 3.11$ & $339.38 \pm 6.03$ & $363.13 \pm 2.55$ & $364.64 \pm 3.93$ & $395 \pm 6.28$ \\
\hline
\end{tabular}

Values are presented as mean \pm standard error. 


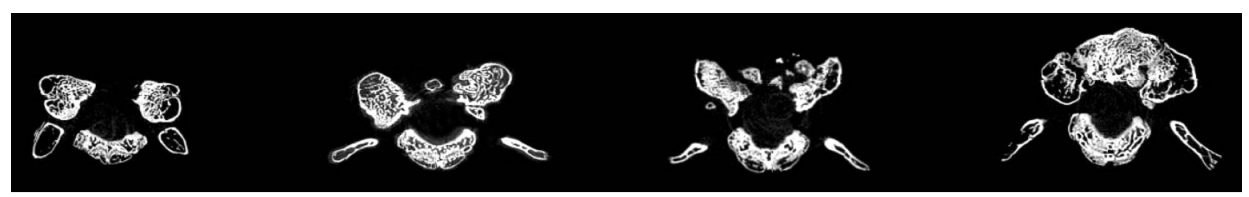

(A) OVX $\quad$ Alendronate $\quad$ Curcumin $\quad$ Alendronate+curcumin

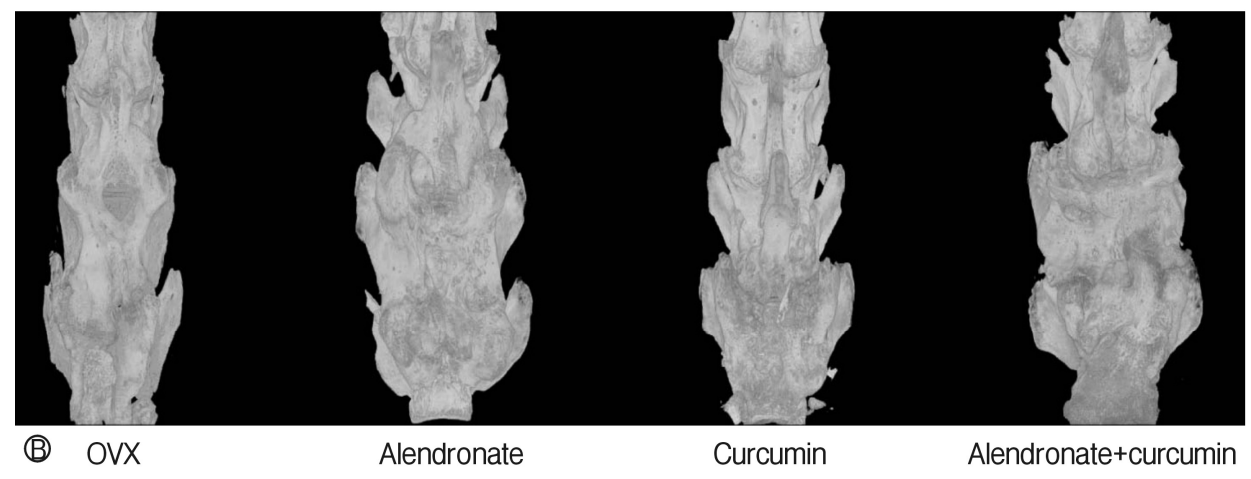

Fig. 2. Representative micro-computed tomography (CT) images of the fused lumbar spine. (A) Axial CT images. (B) Three-dimensional reconstruction images. OVX, ovariectomy.

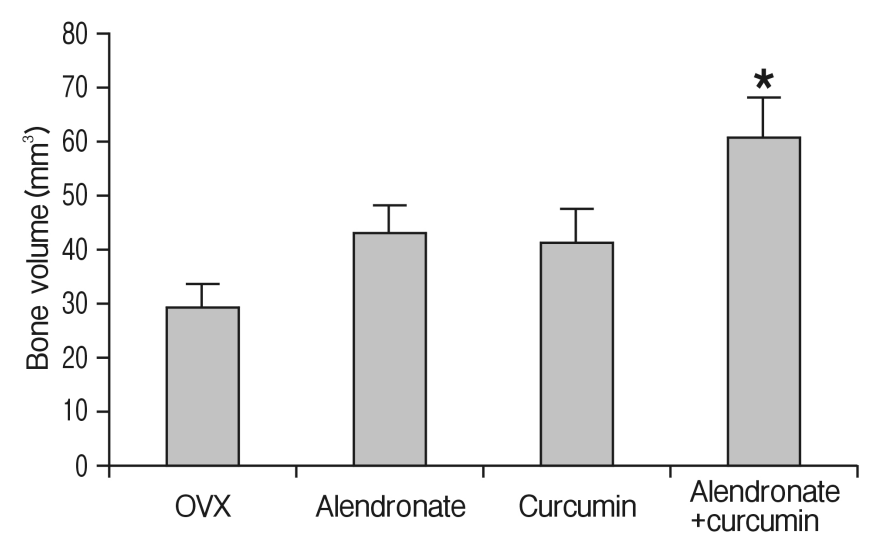

Fig. 3. Graph showing fusion volume after 8 weeks after spine fusion surgery. The combination therapy group had a significantly higher fusion volume compared with the curcumin-only group. " $\mathrm{p}<0.05$ for the combination therapy group vs. the curcumin-only group. OVX, ovariectomy.

\section{Bone Fusion Volume}

As shown in the representative micro-CT images of the fused lumbar spine (Fig. 2), 8 weeks after fusion surgery, the fusion masses comprised a mixture of dispersed grafted bone fragments and newly formed bone. The alendronate-only and the curcumin-only groups had a tendency towards higher fusion volume compared than with the OVX group, though these findings were not statistically significant $(p>0.05)$. However, fusion volume in combination therapy group was significantly higher than in the OVX group $(\mathrm{p}=0.002)$. In the radiographic study and micro-CT scanning, the combination therapy group had a trend towards higher fusion volume at 8 weeks after spine fusion surgery com-

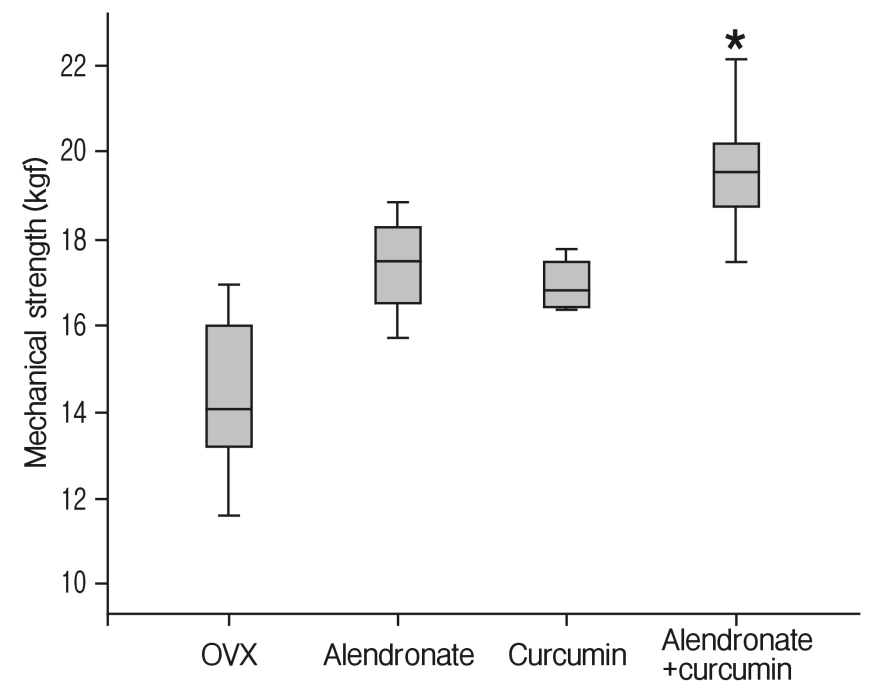

Fig. 4. The 3-point bending test showing the loading force to the maximal load on the fusion mass in L4 and L5 vertebra. * $p<0.05$ for the values of the combination therapy group compared with that of the curcumin-only group. OVX, ovariectomy.

pared with alendronate-only group $(\mathrm{p}=0.053)$. And the combination therapy group had a significant increase in fusion volume at 8 weeks after spine fusion surgery compared with curcuminonly group $(\mathrm{p}=0.039)$ (Fig. 3).

\section{Mechanical Strength}

Biomechanical testing was performed to measure the strength of the fused bone mass. The alendronate-only and the curcuminonly groups had higher mechanical strength compared than with the OVX group, which were statistically significant $(p=0.008$ 
and $p=0.02$, respectively). The 3-point bending test showed that the fused 4th and 5th lumbar vertebrae of the combination therapy group had a trend towards higher mechanical strength compared with alendronate-only group $(p=0.053)$. And combination therapy group had a significantly greater maximal load value compared to that of curcumin-only group $(\mathrm{p}=0.024)$ (Fig. 4).

\section{DISCUSSION}

To our knowledge, the present study is the first study to report the effect of the combination therapy of alendronate and curcumin on spine fusion in a rat model. This study demonstrated that the combination therapy of alendronate and curcumin had statistically significant therapeutic advantages in improving fusion volume and bone strength in the osteoporosis rat spine fusion model.

As a large portion of patients undergoing spine surgery are elderly, there is a corresponding proportion who are chronically exposed to bisphosphonates ${ }^{3}$. Because successful bone graft healing is achieved through appropriate coordination between bone formation and resorption, the excessive suppression of bone turnover induced by treatment with bisphosphonates can lead to fusion failure after spine fusion surgery. Thus, it is clinically relevant to understand the effects of bisphosphonates on spine fu$\operatorname{sion}^{7,9,17)}$.

However, the role of bisphosphonate on bone fuion after spine fusion surgery remains debatable ${ }^{13,18,22,26)}$. Huang et al. ${ }^{13)}$ studied the effect of alendronate on spine fusion in a rat model. In their study, they demonstrated that manual palpation fusion rates were lower in alendronate-treated groups than in a control group. Fusion masses in alendronate-treated animals were radiographically denser and larger than those in controls, despite lower manual palpation fusion rates. Therefore they recommended patients undergoing spine arthrodesis should not take alendronate until fusion is achieved. On the contrary, Xue et al. ${ }^{26}$ evaluated the influence of alendronate treatment and the significance of different amounts of bone graft on posterolateral spine fusion in a porcine model, finding that alendronate treatment decreased fusion mass remodeling without inhibiting fusion rate. There was no statistically significant difference in either fusion rate or fusion mass volume between the alendronate-treated group and control group. Nakao et al. ${ }^{18)}$ recently studied the influence of alendronate on spine fusion in an osteoporotic rat model. They revealed that alendronate was effective for radiologic, biomechanical, and histologic success of spine fusion in an osteoporotic animal model.

Recently, a prospective randomized trial has been published whether alendronate disturbs the healing process of posterior lumbar interbody fusion in human patients ${ }^{17}$. In their study, bridging bone formation was more frequently observed in the alendronate group throughout the postoperative assessment period. A solid bone fusion was achieved in $95 \%$ of the patients in the alendronate group and $65 \%$ of those in the control group. They suggested that favorable mechanical circumstances provided by alendronate overcame its detrimental biological effect on the healing process of spinal fusion. Therefore they recommended that osteoporosis patients undergoing spinal fusion take bisphosphonates throughout the postoperative period. In the present study, we also achieved higher fusion rates and fusion volume in alendronate-treated group compared than in OVX group, though these were not statistically significant $(p>0.05)$. The present results were consistent with those of recent studies ${ }^{17,18,26)}$

Due to the possible undesirable adverse effects associated with bisphosphonate treatment ${ }^{8,10,25)}$, it is worth studying curcumin which has a similar actions to those of the bisphosphonates in bone remodeling ${ }^{16-18,25)}$. Curcumin, which is found in the rhizomes of the turmeric plant (Curcumin longa L.), has been used for centuries as a dietary spice and as a traditional Indian medicine $^{4,12,14,23)}$. In addition to the diverse effects of curcumin, including anti-inflammatory, antioxidant, antiviral, anti-infectious properties, recent studies revealed the effects of curcumin on the regulation of bone remodeling ${ }^{4,11,12,14,23)}$. Previously, at our institute, we studied the effect of curcumin in ovariectomized rats. Due to the low oral bioavailability of curcumin in rats ${ }^{4,12)}$, we demonstrated therapeutic advantages of treatment of highdose curcumin in the ovariectomized rat ${ }^{5}$. In addition, on the basis of similar actions of curcumin and alendronate, we also demonstrated a synergistic bone sparing effect of combination therapy of curcumin and alendronate in ovariectomized rat, the combination therapy group showed the synergistic antiresorptive effect on bone remodeling and improving mechanical strength ${ }^{6}$.

As mentioned before, although the role of bisphosphonate on bone fusion after spine fusion surgery remain controversial $^{13,18,22,26)}$, recent studies have revealed higher fusion rates and fusion volumes with bisphosphonate after spine fusion surgery ${ }^{17,18,26}$. In addition, there are no reports in the literature regarding the effect of curcumin on spine fusion. Therefore, the present study was designed to evaluate whether combination therapy with alendronate and curcumin promotes bone fusion and mechanical strength in spine fusion surgery. In the study, the solid fusion rate was 50\% in the OVX group and 75\% in the alendronate-only and curcumin-only groups. As expected, the combination therapy group had a higher fusion rate compared with the other groups, though not statistically significantly ( $p>0.05$ ). Thus the present results were consistent with those of previous studies, which showed improving spine fusion healing in the presence of osteoporosis ${ }^{17,18,26}$.

In both the alendronate-only and the curcumin-only group compared with OVX group, we achieved the similar results of bone fusion volume, but a significant increase of mechanical strength. However, the combination therapy group had a significantly higher bone fusion volume and mechanical strenghth compared to those in the curcumin-only group $(\mathrm{p}=0.039$ and $p=0.024$, respectively). These results suggest the possibility that additional treatment of curcumin after spine fusion surgery in the presence of osteoporosis can promote higher fusion volume and mechanical strength.

This study is limited due to small number of subjects and the absence of histomorphometric analysis. Histologic findings in 
the fusion mass area can revealed excellent bone neogenesis around the grafted bone. Nevertheless, the present study has revealed that the combination therapy of alendronate and curcumin has a positive effect in the OVX rat spinal fusion model described here.

\section{CONCLUSION}

The present study demonstrated that additional treatment of curcumin and alendronate after spine fusion surgery in rat can promote higher fusion volume, and improve bone mechanical strength.

\section{CONFLICT OF INTEREST}

No potential conflict of interest relevant to this article was reported.

\section{ACKNOWLEDGMENTS}

This work was supported by Biomedical Research Institute grant, Kyungpook National University Hospital (2013).

\section{REFERENCES}

1. Abe Y, Takahata M, Ito M, Irie K, Abumi K, Minami A: Enhancement of graft bone healing by intermittent administration of human parathyroid hormone (1-34) in a rat spinal arthrodesis model. Bone 41:775-785, 2007

2. Adachi JD, Loannidis G, Berger C, Joseph L, Papaioannou A, Pickard L, et al: The influence of osteoporotic fractures on healthrelated quality of life in community-dwelling men and women across Canada. Osteoporos Int 12:903-908, 2001

3. Babat LB, McLain R, Milks R, Ferrara L, Sohn MJ: The effects of the antiresorptive agents calcitonin and pamidronate on spine fusion in a rabbit model. Spine J 5:542-547, 2005

4. Bharti AC, Takada Y, Aggarwal BB: Curcumin (diferuloylmethane) inhibits receptor activator of NF-kappa B ligand-induced NF-kappa $\mathrm{B}$ activation in osteoclast precursors and suppresses osteoclastogenesis. J Immunol 172:5940-5947, 2004

5. Cho DC, Jung HS, Kim KT, Jeon Y, Sung JK, Hwang JH: Therapeutic advantages of treatment of high-dose curcumin in the ovariectomized rat. J Korean Neurosurg Soc 54:461-466, 2013

6. Cho DC, Kim KT, Jeon Y, Sung JK: A synergistic bone sparing effect of curcumin and alendronate in ovariectomized rat. Acta Neurochir (Wien) 154:2215-2223, 2012

7. Cho JH, Cho DC, Yu SH, Jeon YH, Sung JK, Kim KT: Effect of dietary calcium on spinal bone fusion in an ovariectomized rat model. J Korean Neurosurg Soc 52:281-287, 2012

8. Delmas PD: Treatment of postmenopausal osteoporosis. Lancet 359:2018-2026, 2002

9. Diamond TH, Clark WA, Kumar SV: Histomorphometric analysis of fracture healing cascade in acute osteoporotic vertebral body fractures. Bone 40:775-780, 2007

10. Fleisch H: Bisphosphonates: mechanisms of action. Endocr Rev 19:80-100, 1998

11. Folwarczna J, Zych M, Trzeciak HI: Effects of curcumin on the skeletal system in rats. Pharmacol Rep 62:900-909, 2010

12. French DL, Muir JM, Webber CE: The ovariectomized, mature rat model of postmenopausal osteoporosis: an assessment of the bone sparing effects of curcumin. Phytomedicine 15:1069-1078, 2008

13. Huang RC, Khan SN, Sandhu HS, Metzl JA, Cammisa FP Jr, Zheng F, et al: Alendronate inhibits spine fusion in a rat model. Spine (Phila Pa 1976) 30:2516-2522, 2005

14. Joe B, Vijaykumar M, Lokesh BR: Biological properties of curcumin-cellular and molecular mechanisms of action. Crit Rev Food Sci Nutr 44:97-111, 2004

15. Kashii M, Hashimoto J, Nakano T, Umakoshi Y, Yoshikawa H: Alendronate treatment promotes bone formation with a less anisotropic microstructure during intramembranous ossification in rats. J Bone Miner Metab 26:24-33, 2008

16. Li C, Wang HR, Li XL, Zhou XG, Dong J: The relation between zoledronic acid infusion and interbody fusion in patients undergoing transforaminal lumbar interbody fusion surgery. Acta Neurochir (Wien) 154:731-738, 2012

17. Nagahama K, Kanayama M, Togawa D, Hashimoto T, Minami A: Does alendronate disturb the healing process of posterior lumbar interbody fusion? A prospective randomized trial. J Neurosurg Spine 14:500-507, 2011

18. Nakao S, Minamide A, Kawakami M, Boden SD, Yoshida M: The influence of alendronate on spine fusion in an osteoporotic animal model. Spine (Phila Pa 1976) 36:1446-1452, 2011

19. Park SB, Park SH, Kim NH, Chung CK: BMP-2 induced early bone formation in spine fusion using rat ovariectomy osteoporosis model. Spine J 13:1273-1280, 2013

20. Reginster JY, Burlet N: Osteoporosis: a still increasing prevalence. Bone 38(2 Suppl 1):S4-9, 2006

21. Russell RG, Rogers MJ: Bisphosphonates: from the laboratory to the clinic and back again. Bone 25:97-106, 1999

22. Sama AA, Khan SN, Myers ER, Huang RC, Cammisa FP Jr, Sandhu $\mathrm{HS}$, et al: High-dose alendronate uncouples osteoclast and osteoblast function: a study in a rat spine pseudarthrosis model. Clin Orthop Relat Res (425):135-142, 2004

23. Shishodia S, Sethi G, Aggarwal BB: Curcumin: getting back to the roots. Ann N Y Acad Sci 1056:206-217, 2005

24. Solomon DH, Hochberg MC, Mogun H, Schneeweiss S: The relation between bisphosphonate use and non-union of fractures of the humerus in older adults. Osteoporos Int 20:895-901, 2009

25. Theriault RL, Hortobagyi GN: The evolving role of bisphosphonates. Semin Oncol 28:284-290, 2001

26. Xue Q, Li H, Zou X, Bünger M, Egund N, Lind M, et al: The influence of alendronate treatment and bone graft volume on posterior lateral spine fusion in a porcine model. Spine (Phila Pa 1976) 30:1116-1121, 2005

27. Yee AJ, Bae HW, Friess D, Robbin M, Johnstone B, Yoo JU: Accuracy and interobserver agreement for determinations of rabbit posterolateral spinal fusion. Spine (Phila Pa 1976) 29:1308-1313, 2004 